\title{
Comparison of short-term outcomes of anterolateral supine approach and posterolateral approach for primary total hip arthroplasty: a retrospective study
}

Taku Ukai ${ }^{*}$, Goro Ebihara and Masahiko Watanabe

\begin{abstract}
Background: This study aims to evaluate postoperative pain and functional and clinical outcomes of anterolateral supine (ALS) and posterolateral (PL) approaches for primary total hip arthroplasty.

Materials and methods: We retrospectively examined the joints of 110 patients who underwent primary total hip arthroplasty (THA). The ALS group was compared with the PL group using the pain visual analog scale (VAS) and narcotic consumption as pain outcomes. Functional outcomes included postoperative range of motion (ROM) of hip flexion, day on which patients could perform straight leg raising (SLR), day on which patients began using a walker or cane, duration of hospital stay, rate of transfer, and strength of hip muscles. Clinical outcomes included pre and postoperative Harris Hip Scores.
\end{abstract}

Results: No significant differences were found in the pain VAS scores or narcotic consumption between the two groups. The PL group could perform SLR earlier than the ALS group $(P<0.01)$. The ALS group started using a cane earlier $(P<0.01)$ and had a shorter hospital stay $(P<0.01)$ than the $P L$ group. Degrees of active ROM of flexion at postoperative day (POD) 1 were significantly lower in the ALS group than in the PL group $(P<0.01)$. Regarding hip muscle strength, hip flexion was significantly weaker in the ALS group than in the PL group until 1-month POD $(P<0.01)$. External rotation from 2 weeks to 6 months postoperatively was significantly weaker in the PL group than in the ALS group $(P<0.01)$.

Conclusion: The ALS approach was more beneficial than the PL approach because ALS enabled better functional recovery of the strength of external rotation, improved rehabilitation, and involved a shorter hospital stay.

Level of Evidence: Level IV retrospective observational study.

Keywords: Total hip arthroplasty, Postoperative pain, Functional and clinical outcomes, Anterolateral supine approach, Posterolateral approach

\section{Introduction}

Various approaches are used for total hip arthroplasty (THA) [1-4], and agreement on the best approach remains controversial. The posterolateral (PL) approach

\footnotetext{
*Correspondence: taku115@is.icc.u-tokai.ac.jp
}

Department of Orthopaedic Surgery, Surgical Science, Tokai University

School of Medicine, 143 Shimokasuya, Isehara, Kanagawa 259-1193, Japan is performed extensively worldwide and is the most fundamental approach. However, this approach entails resection of the short external rotators [5]. Thus, studies have reported higher dislocation rates with the PL approach than with other approaches [6-8]. Anterior approaches are divided into the direct anterior approach (DAA) and the anterolateral supine approach (ALS). These approaches have the advantages of being less 
invasive, having low dislocation rates [9], and resulting in early recovery [10] because both approaches take advantage of the intermuscular plane [11-14]. Although DAA is associated with a lower visual analog scale (VAS) score, longer walking distance, and shorter hospital stay than the posterior approach, it is also associated with lateral femoral cutaneous nerve (LFCN) injury $[15,16]$. Previous studies have reported that $23-30 \%$ of patients who underwent THA via DAA experienced numbness of the lateral thigh $[15,16]$. Compared with DAA, ALS rarely causes LFCN injury and can preserve the anterior capsule, iliofemoral ligament, and conjoint tendon. Preserving these soft tissues is important for hip stability and prevents leg-length discrepancy. However, ALS may cause injury of the inferior branch of the superior gluteal nerve and abductor weakness [17-19].

Although many studies have compared various approaches, aspects of muscle strength other than abduction have rarely been evaluated, and to the best of the authors' knowledge, no study has assessed pain and functional and clinical outcomes of these approaches simultaneously. Evaluating muscle strength is particularly necessary to assess the advantages of a surgical approach. This study therefore aimed to evaluate and compare short-term results of PL and ALS for primary THA by simultaneously assessing pain and functional and clinical outcomes.

\section{Materials and methods \\ Patients}

This study retrospectively examined 110 joints of 110 patients who underwent primary THA between May 2017 and January 2020. All procedures were approved by the ethical committee at the author's institution (19R188). One orthopedic surgeon performed all PL procedures, and another orthopedic surgeon performed all ALS procedures. This study only included patients who underwent primary THA and could walk by themselves before operation. Patients who were aged $<40$ years, had prior ipsilateral hip surgery, dementia, or mental impairment, or used a walker or wheelchair before operation were excluded.

\section{Anesthesia and analgesia}

All patients underwent THA under general anesthesia maintained with total intravenous propofol. Acetaminophen $(1000 \mathrm{mg})$, fentanyl $(100 \mu \mathrm{g})$, and metoclopramide $(10 \mathrm{mg})$ were administered at the end of surgery. For postoperative analgesia, oral acetaminophen was administered at a dose of $1800 \mathrm{mg} / \mathrm{d}$ starting on the day after surgery. All patients received a cocktail injection comprising $0.2 \%$ ropivacaine $(150 \mathrm{mg})$, adrenaline $(0.2 \mathrm{mg})$, methylprednisolone $(40 \mathrm{mg})$, and saline $(10 \mathrm{~mL})$ after the placement of the cup and stem component. Additionally, a solution containing $0.2 \%$ ropivacaine $(150 \mathrm{mg})$, adrenaline $(0.2 \mathrm{mg})$, and saline $(10 \mathrm{~mL})$ was injected intramuscularly (into the $\mathrm{m}$. gluteus medius, $\mathrm{m}$. gluteus maximus, and rotators) and into subcutaneous tissues before wound closure. Wound closure was performed with monofilament suture. Postoperative pain was assessed by a Likert-type scale ranging from 0 to 6 ( 0 , none; 1 , very mild; 2 , mild; 3 , moderate; 4 , severe; 5 , very severe; 6 , intolerable), and severe pain was defined as a score $>4$. Patients in severe pain were treated with an intravenous infusion of acetaminophen $(500 \mathrm{mg})$, pentazocine (15 mg), or flurbiprofen (50 mg).

\section{Surgical procedure}

The PL approach was performed in the decubitus position. After the short external rotators were dissected and the femoral heads were removed, a cementless acetabular component (Pinnacle; DePuy Synthes, Leeds, UK) and a cementless proximally porous-coated femoral component (S-ROM; DePuy Synthes, Warsaw, IN, USA) were placed. Head sizes of $32 \mathrm{~mm}$ were used for all patients. After the placement of the acetabular and femoral components, the short external rotators were repaired by suturing with the greater trochanter. The posterior capsule was also sutured. For all patients, weight-bearing was allowed on the day after surgery as tolerated.

The ALS approach was performed in the supine position, and an image intensifier was used. A cementless acetabular component (Continium; Zimmer Biomet, Warsaw, IN, USA) and cementless proximally porouscoated femoral component (Fitmore; Zimmer Biomet, Winterthur, Switzerland) were placed. The vertical iliofemoral ligament, conjoint tendon, and anterior capsule were preserved. Similar to the PL group, weight-bearing for the ALS group was allowed on the day after surgery as tolerated.

\section{Measurement of muscle strength}

The muscle strengths of hip flexion, extension, abduction, adduction, internal rotation, and external rotation were quantified by using a handheld dynamometer (Anima Co., $\mu$-TasF1, Tokyo, Japan). To standardize the measurement, only one person performed muscle strength measurement of the hip. The measurement was performed in a hospital or an examination room, and we performed the measurement before rehabilitation to avoid fatigue. During the measurement of hip flexion, abduction, and adduction, patients were placed in the supine position with the hips and knees in a neutral position. Muscle strengths during hip internal rotation and external rotation were measured with the patients seated and the hips and knees in $90^{\circ}$ flexion. For hip extension, the patients 
were placed in the prone position with the hips and knees in a neutral position. Each subject performed two trials for all examinations, and the highest peak force was used for analysis. The peak force was normalized to each patient's body weight $(\mathrm{N} / \mathrm{kg})$. We measured the muscle strength on the day of admission and at 2 weeks, 1 month, 3 months, and 6 months after the operation.

\section{Implant alignment}

Postoperative cup inclination, cup anteversion, and stem anteversion were evaluated by using computer tomography. Cup inclination in the coronal plane was measured between the transverse axis of the cup and the interteardrop line. Cup anteversion was measured in the axial plane as the angle between the transverse cup axis and the sagittal plane. Stem anteversion was measured in the axial plane relative to the posterior bicondylar plane of the femur [20].

\section{Clinical outcome}

The Harris Hip Score (HHS) [21] was used to evaluate the preoperative and postoperative clinical outcomes. The HHS was evaluated on the day of admission and at 1 month, 3 months, and 6 months after the operation.

\section{Evaluation}

Preoperative mental wellness was evaluated using the Japanese Orthopedic Association Hip Disease Evaluation Questionnaire (JHEQ) [22]. JHEQ is a self-report questionnaire and consists of three categories: pain, activities of daily living, and mental wellness. Each question is scored using a Likert-type scale (0 points, strongly agree; 1 point, agree; 2 points, uncertain; 3 points, disagree; 4 points, strongly disagree). The mental wellness score of JHEQ ranges from 0 to 28 points, with 0 points indicating the lowest level of mental wellness and 28 points indicating the highest level of mental wellness [22] (Table 1).

Pain was evaluated using VAS $(0 \mathrm{~mm}=$ no pain; $100 \mathrm{~mm}=$ worst imaginable pain) and narcotic consumption. The VAS scores were recorded while patients were at rest in the morning. Pain VAS evaluation was conducted from the day of the surgery $(1 \mathrm{~h}$ after surgery) to 7 days after surgery. Narcotic consumption was recorded until postoperative day (POD) 3. Functional outcome was evaluated using the following information: the active range of motion (ROM) of hip flexion, day on which patients were able to perform straight leg raising (SLR), and day on which patients began using a walker or a cane. ROM and SLR were evaluated in the morning. When ROM was evaluated in a supine position, patients attempted to flex their operated hips independently. The SLR test was also conducted in a supine position, and we considered patients' ability to perform SLR as their ability to keep their lower limbs raised for at least $3 \mathrm{~s}$. Furthermore, we evaluated the length of hospital stay and the rate of transfer. Patients were allowed discharge when they were able to ascend and descend stairs with a cane independently. Patients who were not discharged home until POD 28 were transferred to other hospitals.

\section{Statistical analysis}

Student's $t$ test was used to evaluate age, body mass index (BMI), operation time, VAS, the mental wellness score, cup inclination, cup anteversion, stem anteversion, narcotic consumption, muscle strength (i.e., hip flexion, extension, abduction, adduction, internal rotation, and external rotation), ROM, day on which patients were able to perform SLR, day on which patients began using a walker or a cane, duration of hospital stay, and HHS between the PL and ALS groups. Fisher's exact test was also used to evaluate distribution of sex, diagnosis, and rate of transfer to other hospitals. All tests were performed with a significance level of $P<0.05$. Analyses were performed using the SPSS statistical software (version 26; IBM Corp., Armonk, NY, USA).

Table 1 Mental category of the JHEQ

\section{Question}

Because of my hip-joint disease, I feel dissatisfied with my health

My hip-joint condition deeply affects my well-being

Because of my hip-joint disease, I sometimes feel down

Because of my hip-joint disease, it is difficult to actively undertake various things

Because of my hip-joint disease, I notice how others look at me

Because of my hip-joint pain, sometimes participation in local events and neighborhood relationships do not go smoothly for me

Because of my hip-joint disease, I sometimes quarrel with people

JHEQ: Japanese Orthopedic Association Hip Disease Evaluation Questionnaire 


\section{Results}

The PL approach was performed on 63 patients ( 13 male, 50 female) with a mean age of $65 \pm 11.8$ years. The ALS approach was performed on 37 patients (3 male, 34 female) with a mean age of $66.7 \pm 11.1$ years.

There were no significant differences in cup inclination, cup anteversion, or stem anteversion between the two approaches (Table 2). Operation time in the ALS group was significantly longer than that in the PL group $(P=0.00)$ (Table 3$)$. No significant difference was found between the two groups at any point of VAS and with regard to number of narcotics consumed (Table 4). Degrees of active ROM of flexion at POD 1 were significantly lower in the ALS group than in the PL group $(P<0.01)$. The PL group was able to perform SLR earlier than the ALS group $(P<0.01)$. No significant difference was found regarding the day on which patients began using a walker. However, the day on which patients began using a cane was significantly earlier $(P<0.01)$ and hospital stay was significantly shorter $(P<0.01)$ in

Table 2 Postoperative alignment of implants in the PL and ALS groups

\begin{tabular}{llll}
\hline Implant alignments & PL & ALS & P value \\
\hline Cup inclination $\left(^{\circ}\right)$ & $39.4 \pm 7$ & $41.7 \pm 6.3$ & 0.62 \\
Cup anteversion $\left(^{\circ}\right)$ & $24.9 \pm 11.9$ & $26.3 \pm 14.7$ & 0.27 \\
Stem anteversion $\left(^{\circ}\right)$ & $30.4 \pm 10.6$ & $30.8 \pm 15.2$ & 0.98 \\
\hline
\end{tabular}

ALS: anterolateral supine; PL: posterolateral
Table 4 Postoperative pain (VAS scale) and narcotic consumption of patients in the PL and ALS groups

\begin{tabular}{llll}
\hline $\begin{array}{l}\text { VAS at different PODs } \\
\text { and number of narcotics } \\
\text { consumed }\end{array}$ & PL & ALS & P value \\
\hline VAS (POD 0) & $46.9 \pm 28.1$ & $51.2 \pm 28.4$ & 0.47 \\
POD 1 & $40.5 \pm 26.5$ & $35.3 \pm 28$ & 0.36 \\
POD 2 & $35.8 \pm 26.1$ & $32.3 \pm 25.9$ & 0.53 \\
POD 3 & $24.4 \pm 23.5$ & $25.3 \pm 26.1$ & 0.85 \\
POD 4 & $20.5 \pm 22.2$ & $18.2 \pm 21.7$ & 0.63 \\
POD 5 & $17.4 \pm 21$ & $17.5 \pm 19.3$ & 0.97 \\
POD 6 & $12.4 \pm 16.9$ & $16.5 \pm 19.2$ & 0.27 \\
POD 7 & $11.7 \pm 17.2$ & $16 \pm 18.4$ & 0.25 \\
Narcotic consumption (numbers) & $1.7 \pm 1.8$ & $1.8 \pm 2.1$ & 0.51 \\
\hline
\end{tabular}

ALS: anterolateral supine; PL: posterolateral; POD: postoperative day; VAS: visual analog scale

the ALS group than in the PL group. No significant difference was found regarding rate of transfer (Table 5). Until 1 month after operation, hip flexion was significantly weaker in the ALS group than in the PL group $(P<0.01)$ (Fig. 1). No significant difference was found in hip abduction or adduction between the two groups (Fig. 2). From 2 weeks to 6 months postoperatively, hip external rotation strength was significantly lower in the PL group than in the ALS group $(P<0.01)$ (Fig. 3$)$. No significant differences were found in the preoperative or postoperative HHS between the two groups.

Table 3 Demographic data of patients in the PL and ALS groups

\begin{tabular}{llll}
\hline Patient characteristics & PL & ALS & P value \\
\hline Age (years) & $65 \pm 11.8$ & $66.7 \pm 11.1$ & 0.49 \\
Sex & Male to female (13:50) & Male to female (3:34) & 0.06 \\
Diagnosis & OA 55, ION 7, RDC 1 & OA 25, ION 7, RDC 1, RA 4 & 0.11 \\
BMl & $23.9 \pm 2.9$ & $22.4 \pm 3.6$ & 0.06 \\
Preoperative ROM (degrees) & $87.7 \pm 21.7$ & $84.5 \pm 23.4$ & 0.49 \\
Operation time (minutes) & $58.9 \pm 15.7$ & $112 \pm 29.5$ & 0 \\
Preoperative VAS score & $84.7 \pm 14.3$ & $85.3 \pm 18.1$ & 0.86 \\
Preoperative mental wellness score & $7.4 \pm 5.2$ & $7.7 \pm 6.1$ & 0.82 \\
Preoperative muscle strength (N/kg) & & & 0.4 \\
Flexion & $1.6 \pm 0.8$ & $1.5 \pm 0.6$ & 0.53 \\
Extension & $1.6 \pm 0.5$ & $1.6 \pm 0.5$ & 0.5 \\
Abduction & $1.7 \pm 0.5$ & $1.6 \pm 0.6$ & 0.67 \\
Adduction & $1.4 \pm 0.5$ & $1.2 \pm 0.4$ & 0.35 \\
Internal rotation & $1.1 \pm 0.4$ & $1.1 \pm 0.4$ & 0.27 \\
External rotation & $1.1 \pm 0.4$ & & \\
\hline
\end{tabular}

ALS: anterolateral supine; BMI: body mass index; ION: idiopathic osteonecrosis; OA: osteoarthritis; PL: posterolateral; RA: rheumatoid arthritis; RDC: rapidly destructive coxopathy; VAS: visual analog scale 
Table 5 Postoperative functional evaluation of patients in the PL and ALS groups

\begin{tabular}{llll}
\hline Factors for functional evaluation & PL & ALS & P value \\
\hline ROM (POD 1) (degrees) & $55.7 \pm 19.8$ & $42.6 \pm 18.5$ & 0.001 \\
POD 2 & $61.7 \pm 19$ & $55.4 \pm 20.6$ & 0.13 \\
POD 3 & $70 \pm 17.6$ & $63.2 \pm 26.5$ & 0.13 \\
POD 4 & $78.4 \pm 17.5$ & $72.7 \pm 25.9$ & 0.19 \\
POD 5 & $82.8 \pm 17$ & $77.8 \pm 21.8$ & 0.22 \\
POD 6 & $84.8 \pm 17.1$ & $78.6 \pm 19.4$ & 0.1 \\
POD 7 & $86.1 \pm 18.4$ & $81.2 \pm 18.7$ & 0.21 \\
SLR (days until achievement) & $2.5 \pm 2.9$ & $4.3 \pm 3.4$ & 0.006 \\
Walker (days until achievement) & $4.4 \pm 2.3$ & $3.9 \pm 2.1$ & 0.31 \\
Cane (days until achievement) & $9.1 \pm 3.5$ & $7.1 \pm 2.6$ & 0.004 \\
Hospital stay (days) & $18.6 \pm 4.1$ & $15.5 \pm 3.6$ & 0.001 \\
Rate of transfer & $4 / 63$ & $3 / 34$ & 1.0 \\
\hline
\end{tabular}

ALS: anterolateral supine; PL: posterolateral; POD: postoperative day; ROM: range of motion; SLR: straight leg raising

\section{Discussion}

In this study, we compared the outcomes of the PL and ALS approaches among patients who underwent THA. We found no significant difference in pain outcome between the two groups. For functional evaluation, the PL group was able to perform SLR significantly earlier than the ALS group. Until 1 month postoperatively, the strength of hip flexion was significantly weaker in the ALS group than in the PL group. However, the day on which patients began using a cane was significantly earlier, hospital stay was significantly shorter, and from 2 weeks to 6 months postoperatively, the strength of hip external rotation was significantly higher in the ALS group than in the PL group.

Various approaches are used for THA. Each approach has both advantages and disadvantages. Although the PL approach is used extensively worldwide, it has a high risk of dislocation compared with other approaches [23]. On the contrary, the ALS approach has been reported to lead to a low dislocation rate, less postoperative pain, better functioning of the abductor muscle, and a shorter hospital stay [2, 6, 24-26]. However, this approach is correlated with the damage to the femoral shaft and malalignment of the femoral component [27].

With regard to postoperative pain, Wang et al. compared DAA with PL and found that the DAA group reported significantly lower pain intensity at 24,48 , and $72 \mathrm{~h}$ after surgery [28]. In the present study, no significant

\section{Flexion of hip (N/Kg)}

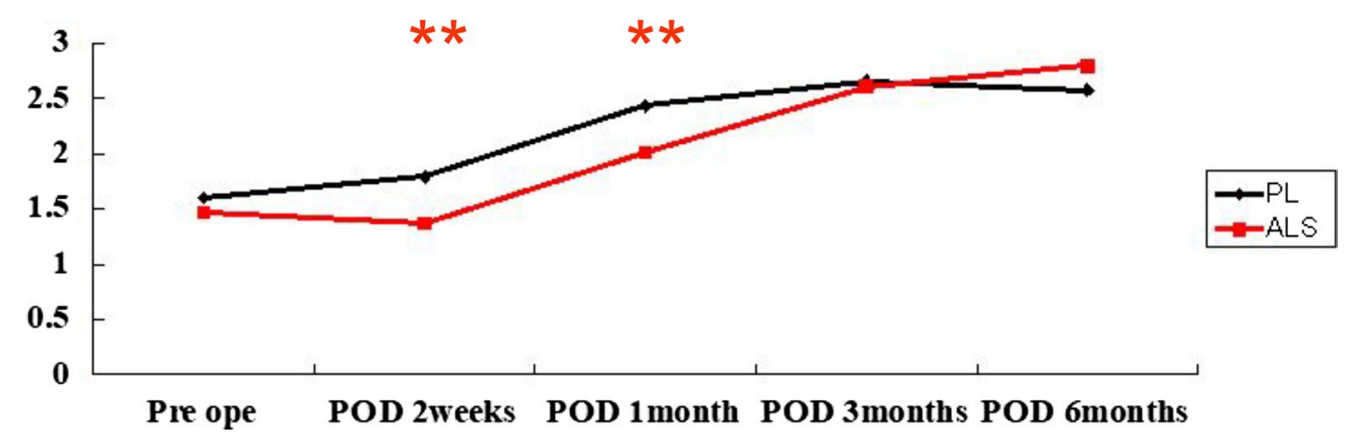

Extention of hip (N/Kg)

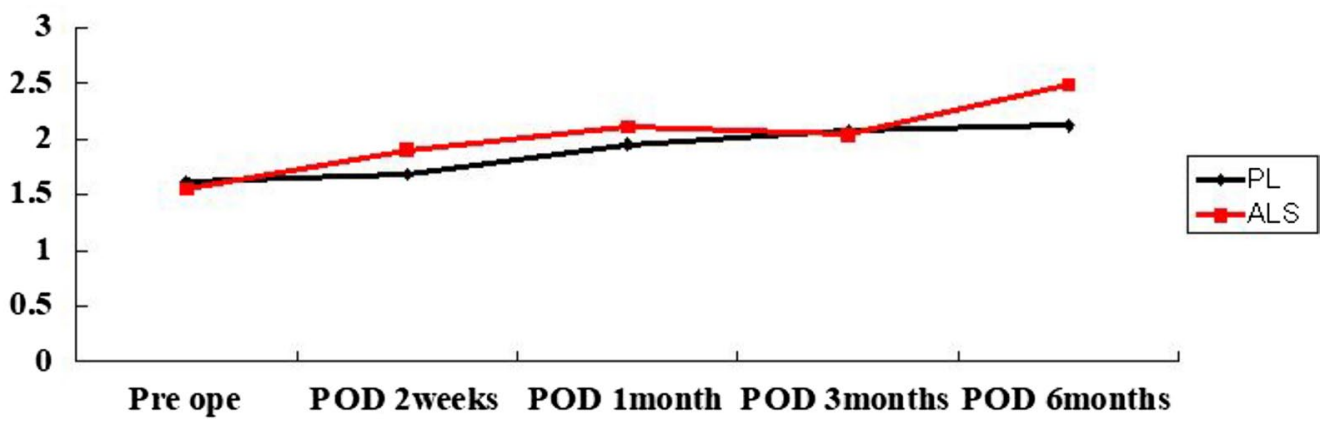

Fig. 1 Evaluation of muscle strength of hip flexion and extension. Hip flexion was significantly weaker in the ALS group than in the PL group until 1 month after operation. ALS: anterolateral supine; PL: posterolateral; POD: postoperative day. ${ }^{*}$ Indicates significance at $P<0.01$ 


\section{Abduction of hip (N/Kg)}

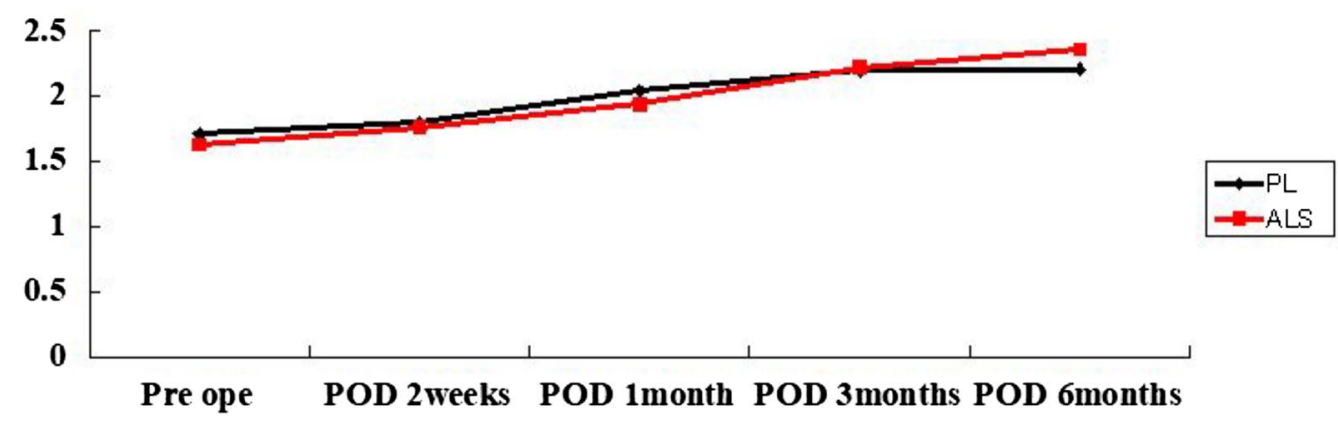

Adduction of hip (N/Kg)

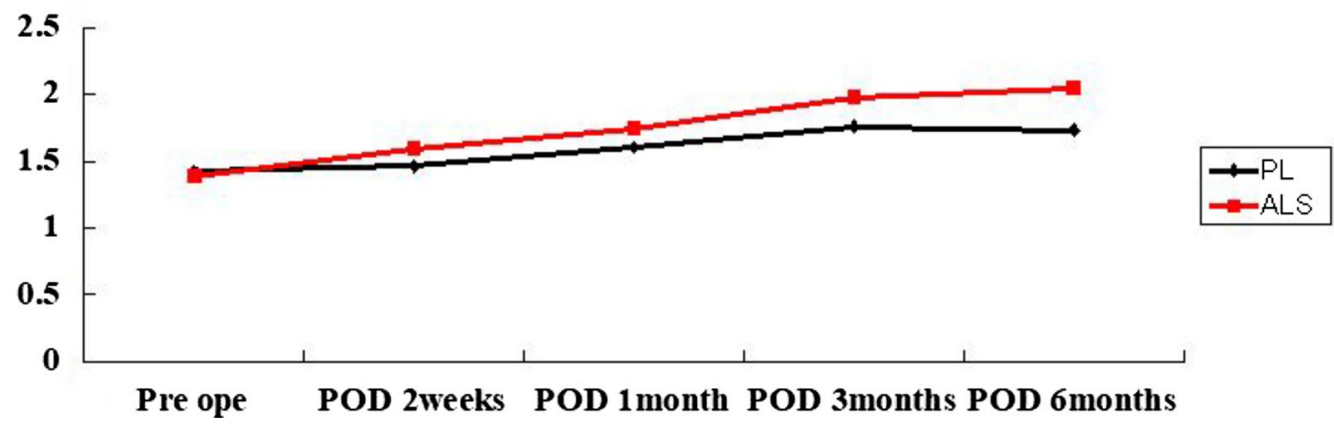

Fig. 2 Evaluation of muscle strength of hip abduction and adduction. No significant difference was found in hip abduction or adduction between the two groups. ALS: anterolateral supine; PL: posterolateral; POD: postoperative day

difference was found in postoperative pain VAS scores between the ALS group and the PL group. We initially considered that, when we conducted the ALS approach, we may have injured the gluteus medius muscle, which could have affected the pain VAS score because the ALS approach has been reported to cause superior gluteal nerve and gluteus medius muscle injuries [17-19]. However, the measurement of the muscle strength during hip abduction revealed no significant difference between the ALS group and the PL group (Fig. 2). In this study, operation time was significantly longer in the ALS group than in the PL group. Even if conventional, the ALS approach tends to require a longer operation time than the $\mathrm{PL}$ approach because the PL approach is one of the most basic approaches. On the other hand, the ALS approach is more difficult to perform than the PL approach, particularly with regard to stem insertion.

The iliofemoral ligament is the strongest ligament of the hip capsule and works to resist the external rotation and extension of the hip [29,30]. Although we initially resected the anterior capsule and iliofemoral ligament, we preserved them in this study to enforce stability of the hip and avoid leg-length discrepancy. However, preserving the iliofemoral ligament entails the restriction of the surgical field and restriction of the elevation of the femur. Due to that, this method tends to require more time for retracting muscles of the anterior hip than the conventional ALS approach. This means that retracting the muscles of the anterior hip may cause swelling of the anterior hip muscles and increase the pain VAS score, especially during the early post-THA period. In fact, patients in the ALS group were able to perform SLR significantly later than patients in the PL group (Table 5).

With regard to the functional outcome, Higgins et al. compared the DAA group and the PL group by assessing estimated blood loss, intraoperative fractures, length of hospital stay, and likelihood of discharge and reported that the DAA group had better outcomes related to length of stay and dislocations [4]. Wang et al. also compared the DAA group and the PL group by assessing the pain VAS scores, incision length, operation time, blood loss, length of hospital stay, and complications, and they reported that the DAA approach was associated with early functional recovery compared with the PL approach [28]. However, they did not assess muscle strength, ROM, or functional outcomes (e.g., SLR and use of a walker or cane). Although these factors are necessary for assessing functional recovery, they are rarely evaluated. Compared 


\section{Internal rotation of hip $(\mathrm{N} / \mathrm{Kg})$}

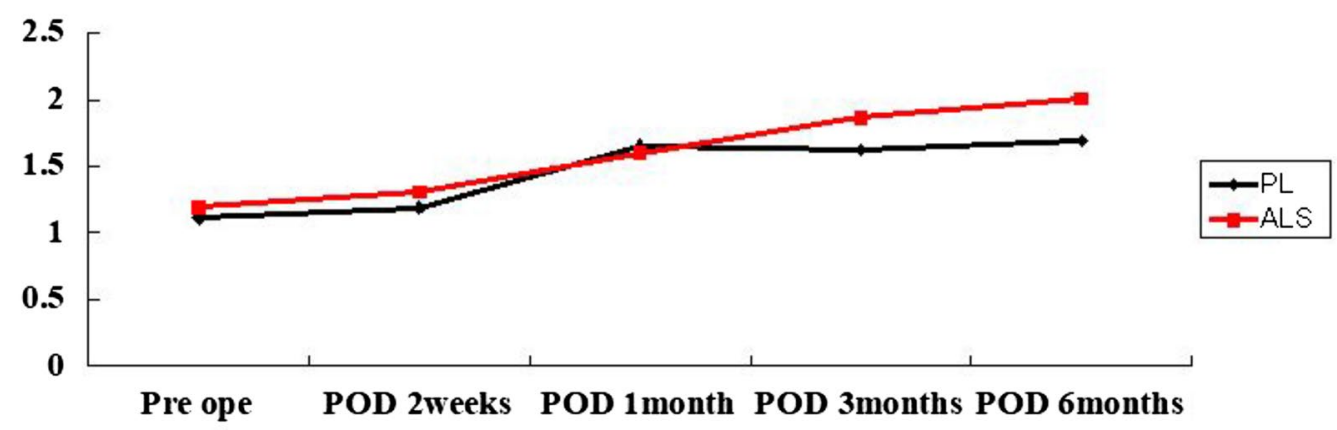

\section{External of hip (N/Kg)}

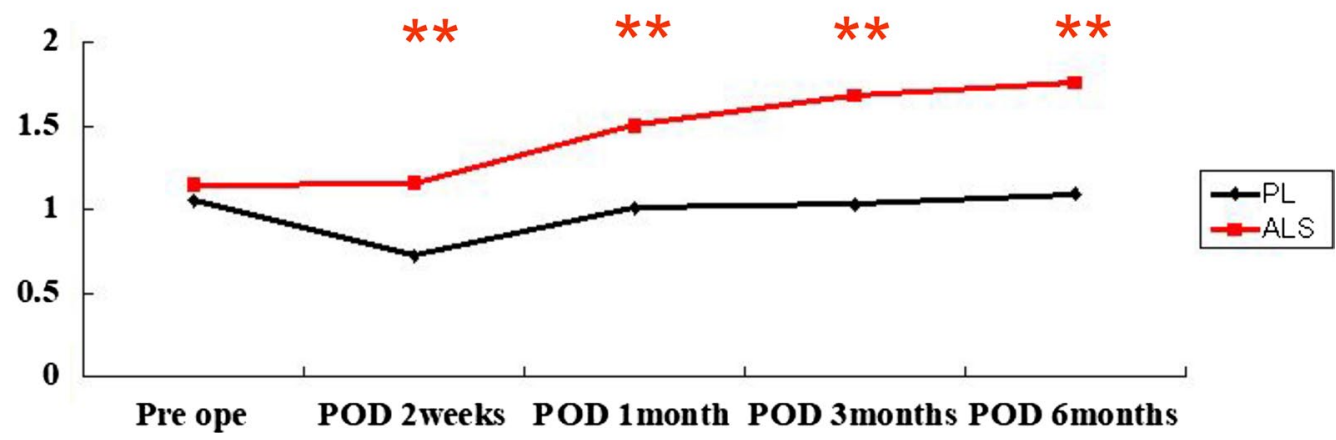

Fig. 3 Evaluation of muscle strength of hip internal and external rotation. From 2 weeks to 6 months postoperatively, hip external rotation strength was significantly lower in the PL group than in the ALS group. ALS, anterolateral supine; PL, posterolateral; POD, postoperative day. ${ }^{* *}$ Indicates significance at $P<0.01$

with the PL approach, the DAA and ALS approaches are considered less invasive because they do not involve the cutting of any muscles. Nevertheless, the extent and duration of motor weakness is still unclear. Although our findings revealed that the ALS approach was correlated with a delay in SLR and weakness of the anterior hip muscles until 1 month postoperatively, we believe that the ALS approach leads to better functional outcomes than the PL approach because weakness in the hip external rotators persisted for up to 6 months after surgery in the PL group. In addition, patients in the ALS group began using a cane earlier than those in the PL group and experienced shorter hospital stays.

Several limitations in this study should be noted. First, this study had a retrospective design. Second, the ALS and PL approaches were not performed by the same surgeons, which may have affected functional outcomes. However, they each had extensive experience as a hip surgeon ( $>10$ years, $>100$ cases). Additionally, both surgeons participated in all operations, and surgical procedures were unified. Third, we did not assess motion pain after rehabilitation. We did initially attempt to assess motion pain after THA, but standardizing the assessment of motion pain proved to be too difficult because
ROM of the hip and progress of rehabilitation varied according to each individual. For instance, the degree of motion pain after gait training compared with that after rehabilitation in bed are quite different. Fourth, muscle strength was measured by only one person who was not blinded and there was no mediator, which may have biased the results. However, although it is possible for the measurement of muscle strength to be highly variable, the tester has had sufficient experience with a handheld dynamometer ( $>5$ years and $>100$ patients). Fifth, each approach was performed with different implants, and only the ALS approach used intraoperative image intensifiers. Although there is no obvious evidence that intraoperative image intensifiers or different implant systems directly influence postoperative pain, rehabilitation, or muscle strength, different implant systems can influence the malalignment of implants, and some studies have reported that implant alignment differed by approach $[31,32]$. There is also a possibility that implant alignment affects muscle tension and gait, as asserted by Tsai et al. who reported that cup anteversion influenced the hip kinematics during gait [33]. However, there were no significant differences in cup inclination, cup anteversion, or stem anteversion between the two approaches in 
the present study (Table 2). Thus, we do not believe that intraoperative image intensifiers or different implant systems affected the results of this study. Finally, the length of operation time was significantly different between the ALS and PL groups, which may have affected pain VAS scores and functional outcomes. In the future, we plan to investigate the short-term results between the ALS and PL approaches by conducting a randomized controlled study.

In conclusion, the ALS approach was correlated with weakness of the anterior hip muscles during the early post-THA period. However, functional outcomes of the ALS group were better than those of the PL group because ALS enabled a better functional recovery of the strength of external rotation, improved rehabilitation, and resulted in shorter hospital stays.

\section{Abbreviations}

ALS: Anterolateral supine; BMI: Body mass index; DAA: Direct anterior approach; JHEQ: Japanese Orthopedic Association Hip Disease Evaluation Questionnaire; HHS: Harris Hip Score; LFCN: Lateral femoral cutaneous nerve; PL: Posterolateral; POD: Postoperative day; ROM: Range of motion; SLR: Straight leg raising; THA: Total hip arthroplasty; VAS: Visual analog scale.

\section{Acknowledgements}

None.

\section{Authors' contributions}

TU designed the study; TU and GE collected data; TU was the major contributors to writing the manuscript. All authors read and approved the final manuscript

\section{Funding}

None.

\section{Availability of data and materials}

Not applicable.

\section{Ethics approval and consent to participate}

This study was approved by the ethics committee of Tokai University. The authors state that this study conforms to the ethical standards laid down in the most recent version of the Declaration of Helsinki. All patients were informed about the study and consented to participate.

\section{Consent for publication}

All patients gave consent for publication.

\section{Competing interests}

The authors declare that there are no conflicts of interest.

Received: 19 September 2020 Accepted: 16 February 2021

Published online: 27 February 2021

\section{References}

1. Sutphen SA, Berend KR, Morris MJ, Lombardi AV (2018) Direct anterior approach has lower deep infection frequency than less invasive direct lateral approach in primary total hip arthroplasty. J Surg Orhtop Adv 27(1):21-24

2. Graves SC, Dropkin BM, Keeney BJ, Lurie JD, Tomek IM (2016) Does surgical approach affect patient-reported function after primary TH A? Clin Orthop Relat Res 474(4):971-981
3. Bernard J, Razanabola F, Beldame J, Driessche SV, Brunel H, Poirier T et al (2018) Electromyographic study of hip muscles involved in total hip arthroplasty: surprising results using the direct anterior minimally invasive approach. Orthop Traumatol Surg Res 104(8):1137-1142

4. Higgins BT, Barlow DR, Heagerty NE, Lin TJ (2015) Anterior vs. posterior approach for total hip arthroplasty, a systematic review and meta-analysis. J Arthroplasty 30(3):419-434

5. Bal BS, Haltom D, Aleto T, Barrett M (2005) Early complications of primary total hip replacement performed with two-incision minimally invasive technique. J Bone Joint Surg Am 87(11):2432-2438

6. Kwon MS, Kuskowski M, Mulhall KJ, Macaulay W, Brown TE, Saleh KJ (2006) Does surgical approach affect total hip arthroplasty dislocation rates? Clin Orthop Relat Res 447:34-38

7. Chiu FY, Chen CM, Chung TY, Lo WH, Chen TH (2000) The effect of posterior capsulorrhaphy in primary total hip arthroplasty: a prospective randomized study. J Arthroplasty 15(2):194-199

8. Sierra RJ, Raposo JM, Trousdale RT, Cabanela ME (2005) Dislocation of primary THA done through a posterolateral approach in the elderly. Clin Orthop Relat Res 441:262-267

9. Demos HA, Rorabeck CH, Bourne RB, MacDonald SJ, McCalden RW (2001) Instability in primary total hip arthroplasty with the direct lateral approach. Clin Orthop Relat Res 393:168-180

10. Malek IA, Royce G, Bhatti SU, Whittaker JP, Phillips SP, Wilson IRB et al (2016) comparison between the direct anterior and posterior approaches for total hip arthroplasty: the role of an 'Enhanced Recovery' pathway. Bone Joint J 98(6):754-760

11. Brend KR, Lombardi AV Jr, Seng BE, Adams JB (2009) Enhanced early outcomes with the anterior supine intermuscular approach in primary total hip arthroplasty. J Bone Joint Surg Am 91(Suppl 6):107-120

12. Kennon RE, Keggi JM, Wetmore RS, Zatorski LE, Huo MH, Keggi LJ (2003) Total hip arthroplasty through a minimally invasive anterior surgical approach. J Bone Joint Surg Am 85(Suppl 4):39-48

13. Parratte S, Pagnano MW (2008) Muscle damage during minimally invasive total hip arthroplasty: cadaver-based evidence that it is significant. Instr Course Lect 57:231-234

14. Meneghini RM, Pagnano MW, Trousdale RT, Hozack WJ (2006) Muscle damage during MIS total hip arthroplasty: Smith-Petersen versus posterior approach. Clin Orthop Relat Res 453:293-298

15. Takada R, Jinno T, Miyatake K, Hirao M, Kimura A, Koga D et al (2018) Direct anterior versus anterolateral approach in one-stage supine total hip arthroplasty. Focused on nerve injury: a prospective, randomized, controlled trial. J Orthop Sci 23(5):783-787

16. Homma Y, Baba T, Sano K, Ochi H, Matsumoto M, Kobayashi H et al (2016) Lateral femoral cutaneous nerve injury with the direct anterior approach for total hip arthroplasty. Int Ortho 40(8):1587-1593

17. Masonis JL, Bourne R (2002) Surgical approach, abductor function, and total hip arthroplasty dislocation. Clin Orthop Relat Res 405:46-53

18. Lachiewicz PF (2011) Abductor tendon tears of the hip: evaluation and management. J Am Acad Orthop Surg 19(7):385-391

19. Valente G, Taddei F, Jonkers I (2013) Influence of weak hip abductor muscles on joint contact forces during normal walking: probabilistic modeling analysis. J Biomech 46(13):2186-2193

20. Arnould A, Boureau F, Benad K, Pasquier G, Migaud H, Girard J (2015) Computed tomography evaluation of hip geometry restraion after total hip resurfacing. Orthop Traumatol Surg Res 101(5):571-575

21. Harris WH (1969) Traumatic arthritis of the hip after dislocation and acetabular fractures: treatment by mold arthroplasty. An end-result study using a new method of result evaluation. J Bone Joint Surg Am 51(4):737-755

22. Matsmoto T, Kaneuji A, Hiejima Y, Sugiyama $H$, Akiyama $H$, Atsumi T et al (2012) Japanese orthopaedic association hip disease evaluation questionnaire (JHEQ): a patient-based evaluation tool for hip-joint disease. J Orthop Sci 17:25-38

23. Zijlstra WP, De Hartog B, Van Streenbergen LN, Scheurs BW, Nelissen RGHH (2017) Effect of femoral head size and surgical approach on risk of revision for dislocation after total hip arthroplasty. Acta Orthop 88(4):395-401

24. Berstock JR, Blom AW, Beswick AD (2015) A systematic review and metaanalysis of complications following the posterior and lateral surgical approaches to total hip arthroplasty. Am R Coll Surg Engl 97(1):11-16 
25. Wesseling M, Meyer C, Corten K, Simon JP, Desloovere K, Jonkers I (2016) Does surgical approach or prosthesis type affect hip joint loading one year after surgery? Gait Posture 44:74-82

26. Delgado FG, Broch A, Reina F, Ximeno L, Torras D, García F et al (2013) MAASH technique for total hip arthroplasty: a capsular work. HSS J 9(2):171-179

27. Bernasek TL, Lee WS, Lee HJ, Lee JS, Kim KH, Yang JJ (2010) Minimally invasive primary THA: anterolateral intermuscular approach versus lateral transmuscular approach. Arch Orthop Trauma Surg 130(11):1349-1354

28. Wang Z, Hou JZ, Wu CH, Zhou YJ, Gu XM, Wang HH et al (2018) A systematic review and meta-analysis of direct anterior approach versus posterior approach in total hip arthroplasty. J Orthop Surg Res 13(1):229

29. Myers CA, Register BC, Lertwanich P, Ejinisman L, Pennington WW, Giphart JE et al (2011) Role of the acetabular labrum and the iliofemoral ligament in hip stability: an in vitro biplane fluoroscopy study. Am J Sports Med 39(Suppl):85S-91S

30. Martin HD, Savage A, Braly BA, Palmer IJ, Beall DP, Kelly B (2008) The function of the hip capsular ligaments: a quantitative report. Arthroscopy 24(2):188-195
31. Migliorini F, Eschweiler J, Trivellas A, Rath B, Driessen A, Tingart M et al (2020) Implant positioning among the surgical approaches for total hip arthroplasty: a Bayesian network meta-analysis. Arch Orthop Trauma Surg 140(8):1115-1124

32. Innmann MM, Streit MR, Kolb J, Heiland J, Parsch D, Peter R et al (2015) Influence of surgical approach on component positioning in primary total hip arthroplasty. BMC Musculoskelet Disord 5(16):180

33. Tsai TY, Li JS, Dimitriou D, Kwon YM (2015) Does component alignment affect gait symmetry in unilateral total hip arthroplasty patients? Clin Biomech 30(8):802-807

\section{Publisher's Note}

Springer Nature remains neutral with regard to jurisdictional claims in published maps and institutional affiliations.

\section{Submit your manuscript to a SpringerOpen ${ }^{\circ}$ journal and benefit from:}

- Convenient online submission

- Rigorous peer review

- Open access: articles freely available online

- High visibility within the field

- Retaining the copyright to your article

Submit your next manuscript at $\gg$ springeropen.com 